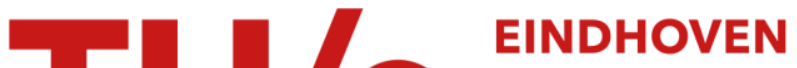 UNIVERSITY OF TECHNOLOGY
}

\section{Energy management for vehicle power net with flexible electric load demand}

\section{Citation for published version (APA):}

Kessels, J. T. B. A., Bosch, van den, P. P. J., Koot, M. W. T., \& Jager, de, A. G. (2005). Energy management for vehicle power net with flexible electric load demand. In Proceedings of the 2005 IEEE Conference on Control Applications (CCA 2005), 28-31 August 2005, Toronto, Canada (pp. 1504-1509). Institute of Electrical and Electronics Engineers. https://doi.org/10.1109/CCA.2005.1507345

DOI:

10.1109/CCA.2005.1507345

Document status and date:

Published: 01/01/2005

\section{Document Version:}

Publisher's PDF, also known as Version of Record (includes final page, issue and volume numbers)

\section{Please check the document version of this publication:}

- A submitted manuscript is the version of the article upon submission and before peer-review. There can be important differences between the submitted version and the official published version of record. People interested in the research are advised to contact the author for the final version of the publication, or visit the $\mathrm{DOI}$ to the publisher's website.

- The final author version and the galley proof are versions of the publication after peer review.

- The final published version features the final layout of the paper including the volume, issue and page numbers.

Link to publication

\section{General rights}

Copyright and moral rights for the publications made accessible in the public portal are retained by the authors and/or other copyright owners and it is a condition of accessing publications that users recognise and abide by the legal requirements associated with these rights.

- Users may download and print one copy of any publication from the public portal for the purpose of private study or research.

- You may not further distribute the material or use it for any profit-making activity or commercial gain

- You may freely distribute the URL identifying the publication in the public portal.

If the publication is distributed under the terms of Article $25 \mathrm{fa}$ of the Dutch Copyright Act, indicated by the "Taverne" license above, please follow below link for the End User Agreement:

www.tue.nl/taverne

Take down policy

If you believe that this document breaches copyright please contact us at:

openaccess@tue.nl

providing details and we will investigate your claim. 


\section{Energy Management for Vehicle Power Net with Flexible Electric Load Demand}

\author{
J.T.B.A. Kessels, P.P.J. van den Bosch \\ Department of Electrical Engineering \\ Technische Universiteit Eindhoven \\ P.O. Box 513, 5600 MB Eindhoven \\ The Netherlands \\ J.T.B.A.Kessels@tue.nl
}

\author{
Michiel Koot, Bram de Jager \\ Department of Mechanical Engineering \\ Technische Universiteit Eindhoven \\ P.O. Box 513, 5600 MB Eindhoven \\ The Netherlands \\ M.W.T.Koot@tue.nl
}

\begin{abstract}
The electric power demand in road vehicles increases rapidly and to supply all electric loads efficiently, energy management (EM) turns out to be a necessity. In general, EM exploits the storage capacity of a buffer connected to the vehicle's power net, such that energy is stored or retrieved at moments that the production of electric power is relative cheap or expensive, respectively. This paper adds an extra degree of freedom, by considering electric loads with a flexible power demand. A strategy based on optimization techniques as well as a rule-based strategy are developed. Simulations illustrate the benefits of applying these flexible loads, as they offer more freedom for EM and moreover, reduce the activity of the storage buffer significantly.
\end{abstract}

\section{INTRODUCTION}

The electric power consumption in road vehicles is increasing rapidly, due to high standards on safety and comfort. Moreover, the introduction of Hybrid Electric Vehicles (HEV), where the propulsion power is partly delivered by an electric machine, contributes to even higher electric power demands. To limit the associated fuel consumption, researchers have proposed smart strategies on how to generate the required electric power [5-8]. Typically, these strategies utilize the storage capacity of a battery at moments when the power requested by the electric loads versus the power of the electric machine is not balanced. This concept has two disadvantages: first, temporarily storing energy always brings additional losses and second, the storage device wears out much faster.

To overcome both problems, this paper considers electric loads with a flexible power demand, such that the requested load power can be adapted to the generated power. Loads with a flexible power demand are characterized by the fact that they accept, up to a certain level, more or less power, without serious performance degradation for the driver. Especially heating and cooling functions are suited for this purpose. The value of this concept is already shown in [1] for an HEV, where the focus is on extra improvements in fuel economy. The aim of this paper is to introduce an Energy Management (EM) strategy for vehicles with a conventional drive train and where the design procedure offers freedom in selecting a trade-off between extra profits on fuel economy versus a reduction in battery wear.

This paper is organized as follows. Section II presents the vehicle model that is used for analyzing strategies. The actual control problem is formulated in Section III. By means of optimization techniques, Section IV presents an analytic control law. Also a rule-based strategy is derived and is explained in Section V. Simulation results are shown in Section VI and the conclusions are given in Section VII.

\section{VEHICLE MODEL}

The scope of this paper is limited to road vehicles with a conventional drive train and a transmission with fixed gear ratios. Modifications are done for the electric power net, where the alternator and parts of the electric loads are replaced by power controlled components. An overview of all relevant power flow signals is given in Fig. 1. In this diagram, the combustion engine converts fuel into mechanical power $P_{m}$. This power is used by the drive train for vehicle propulsion $\left(P_{d}\right)$, whereas a smaller amount of power is used by the alternator $\left(P_{g}\right)$. The electric power $P_{e}$ that comes out of the alternator flows directly to the electric loads $\left(P_{L}\right)$ or is stored in the battery $\left(P_{b}\right)$.

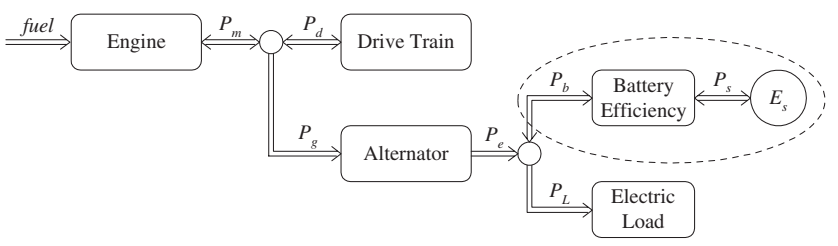

Fig. 1. Overview of vehicle power flow

It is demanded that the driver experiences identical vehicle behavior whether EM is applied or not. Given a driving cycle with a predefined speed profile, one can calculate the power to the drive train as well as the engine speed $\omega$. Consequently, the assumption that the drivability of the vehicle remains unaffected, reduces the complexity of the vehicle model significantly.

An EM strategy exploits the characteristics of the engine, alternator, battery, and electric loads. Except for the battery, quasi-static models have been used for these components. The engine model is described by a nonlinear static map which specifies the relation between fuel consumption fuelrate, engine power $P_{m}$, and engine speed $\omega$ :

$$
\text { fuelrate }=f\left(P_{m}, \omega\right) \text { where } P_{m}=P_{d}+P_{g}
$$


In literature, fuel maps are often presented as a function of engine torque and engine speed. However, the engine torque can be derived from the engine power if the engine speed is known, so these maps represent identical information.

Using a similar approach, the alternator model is captured by a nonlinear static map, expressing the mechanical power $P_{g}$ as a function of the electric power $P_{e}$ :

$$
P_{g}=g\left(P_{e}, \omega\right) \quad \text { where } P_{e}=P_{L}+P_{b}
$$

Obtaining an accurate model of the battery is part of ongoing research. In this paper, the battery model consists of two blocks. The first block introduces the energy losses between the power $P_{b}$ at the battery terminals and the net stored/retrieved power $P_{s}$ :

$$
P_{b}=P_{\text {loss }}\left(P_{s}\right)
$$

For simplicity, the losses in the battery depend only on the actual battery power, but it is very well possible to extend this model with additional parameters such as the actual energy level in the battery or its temperature. More details about the function $P_{\text {loss }}\left(P_{s}\right)$ will be given in Section IV, as this function directly influences the control actions of a strategy. The second block in the battery model keeps track of the energy level $E_{s}$ by means of a simple integrator:

$$
E_{s}(t)=E_{s}(0)+\int_{0}^{t} P_{s}(\tau) d \tau
$$

It is assumed that the energy capacity $E_{c a p}$ of the battery is fixed. Consequently, the relative energy level in the battery can be denoted by the quantity State of Charge:

$$
S O C(t)=\frac{E_{s}(t)}{E_{c a p}} \cdot 100 \%
$$

\section{PROBLEM DEFINITION}

The key idea behind EM is to increase the energy efficiency in the vehicle such that the fuel consumption (and/or tail-pipe emission) is reduced. In this research, this is achieved by moving the operating point of the alternator and hence, the combustion engine to an area where less energy losses are induced. Consequently, the power delivered by the alternator and the power requested by the electric loads might be unbalanced. In that situation, one can either use the storage capacity of the battery or change the power demand of the electric loads. It is clear that rescheduling of the load demand is more profitable than temporarily storing energy in the battery. However, freedom to assign more or less power to the loads is limited.

\section{A. Control objective and constraints}

Following the approach of Tate and Boyd in [9], the concept of EM can be formulated as a (non-linear) optimization problem:

$$
\min _{x} J(x) \quad \text { subject to } \quad G(x) \leq 0
$$

The cost function $J$ is selected such that it represents the vehicle's fuel use over a predefined driving cycle or random trajectory with time interval $t=\left[0, t_{e}\right]$ :

$$
J=\operatorname{fuel}\left(P_{s}, P_{L}\right)=\int_{0}^{t_{e}} \text { fuelrate }\left(P_{s}, P_{L}\right) d t
$$

Note that the design variables are the internal battery power $P_{s}$ and the power to the electric loads $P_{L}$. Using the relations (2) and (3) results in the corresponding setpoint for the alternator power $P_{e}$.

Constraints on the design variables are caused by physical limitations of components as well as requirements with respect to energy conservation. The operating ranges of the engine, alternator, and battery are limited, so inequality constraints are introduced on the minimum and maximum power flow of these components:

$$
\begin{array}{rlrl}
P_{\text {man }} \leq P_{m} \leq P_{\max } & & \forall t \in\left[0, t_{e}\right] \\
P_{\text {emin }} \leq P_{e} \leq P_{\text {emax }} & & \forall t \in\left[0, t_{e}\right] \\
P_{b \min } \leq P_{b} \leq P_{b \max } & \forall t \in\left[0, t_{e}\right]
\end{array}
$$

Furthermore, battery draining has to be prevented. A chargesustaining strategy is obtained by including an end-point constraint on the energy level of the battery:

$$
E_{s}(0)+\int_{0}^{t_{e}} P_{s}(\tau) d \tau \geq E_{\text {sref }}
$$

where $E_{\text {sref }}$ is an arbitrarily selected reference value that should be satisfied at $t=t_{e}$, e.g. $E_{s}(0)$.

Finally, constraints on $P_{L}$ are used to characterize the energy and power demand of the electric loads. It is assumed that all individual loads can be aggregated and result in separate power and energy constraints:

$$
\begin{array}{cc}
P_{L \text { min }} \leq P_{L} \leq P_{L \text { max }} & \forall t \in\left[0, t_{e}\right] \\
\int_{0}^{t} P_{L}(\tau) d \tau \geq E_{L \text { min }}(t) & \forall t \in\left[0, t_{e}\right]
\end{array}
$$

\section{QUADRATIC PROGRAMMING}

Finding the optimal solution for the problem defined in the previous section will be computationally demanding. To come to a solution close to the global optimal solution, the original problem is approximated with a Quadratic Programming (QP) problem. Such a QP-structure is characterized by a quadratic cost function, subject to linear constraints:

$$
\min _{x} \frac{1}{2} x^{\top} H x+h^{\top} x \text { subject to } A x \leq b
$$

\section{A. Model reduction}

To derive a quadratic description for the cost function (7), the models of the individual components need to be reduced. For the engine map, a linear approximation will be used:

$$
\text { fuelrate }\left(P_{m}\right) \approx \alpha_{1} P_{m}+\alpha_{0}
$$

The parameters $\alpha_{1}$ and $\alpha_{0}$ are state dependent and are selected such that they represent a local fit of the fuel map in the area $P_{m}=\left[P_{d}, P_{d}+P_{g \max }\right]$ and engine speed $\omega$. In practical situations, the fuel map of an engine is obtained 


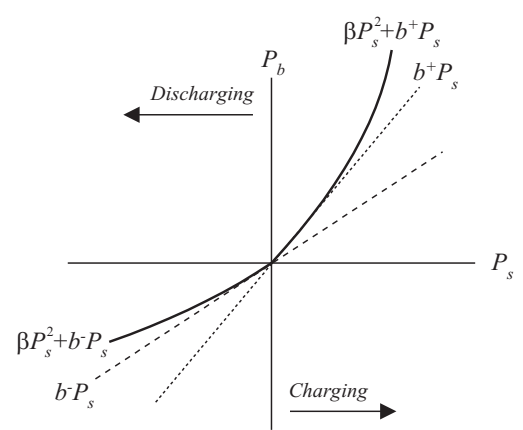

Fig. 2. Parameter selection in battery efficiency model

by measuring its fuel consumption at a finite number of grid points. These grid points cover the entire operating area of the engine. Compared to the power range of the alternator, this is a relative coarse grid and therefore it is acceptable to approximate the fuel consumption by a local linear fit.

Measurement data of the alternator show that energy losses are more than proportional at higher power levels. For that reason, the alternator map is approximated by a quadratic fit:

$$
P_{g}\left(P_{e}\right) \approx \gamma_{2} P_{e}^{2}+\gamma_{1} P_{e}+\gamma_{0}
$$

Again, the parameters $\gamma_{2}, \gamma_{1}$ and $\gamma_{0}$ are state dependent. They approximate the alternator map over its entire power range $P_{e}=\left[P_{e \min }, P_{e \max }\right]$ at a certain engine speed $\omega$.

The dynamic behavior of a battery is rather complex and it is inconvenient to model this accurately in a QP-structure. From a physical point of view, it is reasonable to assume that losses in the battery will increase for higher power flows. Consequently, there has been decided to use a battery model incorporating linear and quadratic losses:

$$
P_{b}\left(P_{s}\right) \approx \beta P_{s}^{2}+\max \left(b^{-} P_{s}, b^{+} P_{s}\right)
$$

In Fig. 2 the contribution of each individual term is shown. The parameter $\beta>0$ represents the quadratic losses whereas $b^{+}>1$ and $0<b^{-}<1$ indicate the piece-wise linear losses during charging and discharging, respectively. Although these losses might not be exact as in reality, they also serve as a tuning parameter to limit the actual battery usage of an EM strategy. That is, incorporating more losses in the battery model than actually present in reality, will discourage any strategy to use the battery as an energy storage buffer. Because battery usage is directly connected to battery wear, the parameters $\beta, b^{+}$and $b^{-}$turn out to be a trade-off between performance of the strategy versus battery wear.

\section{B. $Q P$ formulation}

The battery model described in (17) cannot be directly included in the QP-framework of (14). Fortunately, the restrictions on $\beta, b^{+}$and $b^{-}$guarantee that (17) is always a convex function. As shown in [3], it is possible to reformulate the expression $\max \left(b^{-} P_{s}, b^{+} P_{s}\right)$ as follows:

$$
\min _{P_{a}} P_{a} \text { subject to }\left\{\begin{array}{l}
P_{a} \geq b^{-} P_{s} \\
P_{a} \geq b^{+} P_{s}
\end{array}\right.
$$

Now the substitution of (16)-(18) into (15) results in a 4thorder expression between fuel use and battery power $P_{s}$. Because a quadratic relation is needed for a QP-structure, a second order Taylor approximation has been applied, leaving out the higher order terms. To that end, the design variable $P_{L}$ has changed into the zero-mean variable $\Delta P_{L}$, representing the deviation from the average load power $\tilde{P}_{L}$ :

$$
P_{L}:=\tilde{P}_{L}+\Delta P_{L}
$$

The cost criterion in (7) is written in discrete time with sampling interval $\Delta T$ over $N_{p}$ periods:

$$
J=\sum_{k=1}^{N_{p}} \text { fuelrate }\left(P_{s}(k), \Delta P_{L}(k), P_{a}(k)\right) \Delta T
$$

The selected design-variable $x=\left[\begin{array}{lll}P_{s} & \Delta P_{L} & P_{a}\end{array}\right]^{\top} \in \mathbb{R}^{3 N_{p}}$ covers all periods $k=1, . ., N_{p}$ and results in the following description for $H$ and $h$ in (14):

$$
\begin{gathered}
H=\left[\begin{array}{ccc}
2 \alpha_{1} \gamma_{1} \beta+4 \alpha_{1} \gamma_{2} \beta \tilde{P}_{L} & 0 & 0 \\
0 & 2 \alpha_{1} \gamma_{2} & 2 \alpha_{1} \gamma_{2} \\
0 & 2 \alpha_{1} \gamma_{2} & 2 \alpha_{1} \gamma_{2}
\end{array}\right] \\
h=\left[\begin{array}{c}
0 \\
\alpha_{1} \gamma_{1}+2 \alpha_{1} \gamma_{2} \tilde{P}_{L} \\
\alpha_{1} \gamma_{1}+2 \alpha_{1} \gamma_{2} \tilde{P}_{L}
\end{array}\right]
\end{gathered}
$$

All constraints given in (8)-(10) will be written as linear constraints on $P_{s}$ and $\Delta P_{L}$. By using the inverse relation of (16) and selecting the correct solution, it is possible to write (8) as a constraint on $P_{e}=P_{b}+P_{L}$. Also (9) appears as a constraint on $P_{b}+P_{L}$. Unfortunately, the selected battery model in (17) gives no opportunity to write both constraints as a linear combination of $P_{s}$ and $\Delta P_{L}$. To circumvent this problem, the energy losses in the battery are neglected during constraint handling and $P_{b}$ becomes equivalent to $P_{s}$. A feasible solution for the original constraints in (8)-(10) is guaranteed by slightly reducing the power limits $P_{\max }$, $P_{\text {emax }}$ and $P_{b \max }$. Finally, the three new constraints are combined into one constraint for every period $k=1, . ., N_{p}$ :

$$
P_{e \min }^{*} \leq P_{s}+\tilde{P}_{L}+\Delta P_{L} \leq P_{\text {emax }}^{*}
$$

The end-point constraint in (11) on the energy level in the battery becomes in discrete time:

$$
E_{s}(0)+\sum_{k=1}^{N_{p}} P_{s}(k) \Delta T \geq E_{\text {sref }}
$$

The requirements on load power and energy to the loads are written in terms of $\tilde{P}_{L}+\Delta P_{L}$. For the constraint in (12) this is rather straight forward:

$$
P_{L \min } \leq \tilde{P}_{L}+\Delta P_{L} \leq P_{L \max }
$$

The energy constraint in (13) needs to be evaluated in all periods $k=1, . ., N_{p}$, resulting in $N_{p}$ constraints:

$$
\sum_{i=1}^{k}\left(\tilde{P}_{L}+\Delta P_{L}\right) \Delta T \geq E_{L \min }(k)
$$




\section{Model Predictive Control}

The optimization problem formulated above requires that the entire driving cycle is known in advance. In realworld driving situations, this will be practically impossible. However, the idea that the vehicle speed can be predicted in the near future is certainly realistic. With only minor changes, it is possible to put the QP-problem into a Model Predictive Control (MPC) framework, see [2] and [5]. Instead of performing the optimization in (20) over the entire driving cycle, it will be limited to a prediction horizon of $N_{p}$ periods. Only the first value of the resulting control sequence is implemented, whereas the calculations are repeated each time instant with updated state and prediction information.

\section{RULE-BASED STRATEGY}

The presented MPC-strategy is a feasible solution for online implementation in a vehicle. Nevertheless, the actual performance of this strategy heavily depends on the quality and length of the prediction information. This section presents a rule-based control strategy, with the control law derived in an intuitive way. Robustness is guaranteed, as this strategy does not rely on prediction information.

\section{A. Strategy concept}

Suppose that the generation of $\Delta P_{e}$ electric power results in $\Delta$ fuelrate extra fuel consumption. From this information, the incremental cost $\lambda$ can be defined as the slope of the joined fuel map (1) and alternator map (2):

$$
\lambda\left(P_{e}\right)=\frac{\partial \text { fuelrate }\left(P_{e}\right)}{\partial P_{e}} \quad \text { in }[\mathrm{gr} / \mathrm{J}]
$$

Intuitively, it is clear that a possible strategy should generate (extra) electric power when $\lambda$ is small and generate less when $\lambda$ is large. If there exists no freedom in supplying temporarily more or less power to the electric loads, the battery will be used to store or retrieve this electric power. Due to energy losses in the battery, there will be a tradeoff when the profits obtained from generating at a lower incremental cost are higher than the losses induced in the battery. Two parameters will be introduced that characterize this trade-off. The first parameter is the average incremental cost $\lambda_{a v}$, that represents the average value of (27) for a given driving cycle and EM strategy. Secondly, the efficiency of the battery is defined by parameter $\eta$ and expresses the losses that occur during a complete micro-cycle of charging and discharging the battery:

$$
P_{\text {retrieved }}=\eta P_{\text {stored }}
$$

The parameters $\lambda_{a v}$ and $\eta$ will be used to divide the operating range of the alternator in four areas with each their own control law. On average, it is beneficial to store additional energy in the battery if $\lambda\left(P_{e}\right) \leq \sqrt{\eta} \lambda_{a v}$ and retrieval of energy will be beneficial if $\lambda\left(P_{e}\right) \geq \lambda_{a v} / \sqrt{\eta}$. The area in between denotes the operating range where the battery should not be used. Nevertheless, supplying power to the electric loads is profitable as long as $\lambda\left(P_{e}\right)<\lambda_{a v}$, as they accept energy without additional losses.
Similar to the battery parameters in the optimization strategy, the parameter $\eta$ can be used as tuning parameter between strategy performance versus battery wear. Different from the optimization strategy, loads with a flexible power demand are now characterized by the power constraint in (12) and not by the energy constraint in (13).

\section{B. Strategy implementation}

Three switching levels $P_{e, l}, P_{e, a v}$ and $P_{e, u}$ are calculated that characterize the four operating areas of the alternator. To that end, the complete power range $P_{e} \in\left[P_{e \min }, P_{\text {emax }}\right]$ is mapped onto a dense grid. For each grid point, the incremental cost will be calculated and from this set, three power levels are selected:

$$
\begin{aligned}
P_{e, l} & :=\arg \left\{P_{e} \mid \lambda\left(P_{e}\right)=\sqrt{\eta} \lambda_{a v}\right\} \\
P_{e, a v} & :=\arg \left\{P_{e} \mid \lambda\left(P_{e}\right)=\lambda_{a v}\right\} \\
P_{e, u} & :=\arg \left\{P_{e} \mid \lambda\left(P_{e}\right)=\frac{1}{\sqrt{\eta}} \lambda_{a v}\right\}
\end{aligned}
$$

The implementation of the final strategy boils down to the following two control-laws:

$$
\begin{aligned}
P_{L}= & \max \left[P_{L \min }, \min \left[P_{L \max }, P_{e, a v}\right]\right] \\
P_{b}= & \max \left[\min \left[P_{e, l}-P_{L}, P_{\text {emax }}-P_{L}\right], 0\right] \\
& \quad-\max \left[P_{L}-P_{e, u}, P_{L}-P_{\text {emax }}, 0\right]
\end{aligned}
$$

Limitations on the battery power as specified in (10) are not covered in (33) but will be respected afterwards through saturation. To keep the energy level of the battery between certain boundaries, parameter $\lambda_{a v}$ needs to be carefully selected such that the battery is never overcharged or completely drained. In practice, this requires online adaptation of $\lambda_{a v}$ but for a predefined driving cycle, one can rely on a constant value. Typically, a higher value for $\lambda_{a v}$ results in more energy in the battery as there are more situations where it is assumed that power can be generated cheaply. In simulations, this knowledge is used to select a fixed value for $\lambda_{a v}$ that keeps the final energy level of the battery close to the initial energy level. An arbitrarily small deviation can be achieved by selecting $\lambda_{a v}$ through linear regression on simulation results that achieve already a small error for the final energy level. This approach is further discussed in [6] The corresponding fuel consumption is also found through this method and will be used to compare the performance of this strategy with the optimization strategy.

\section{SIMULATION ENVIRONMENT}

\section{A. Simulation model}

Simulations are carried out for a mid-sized vehicle with a 2.0L spark-ignition engine and manual transmission, driving the New European Driving Cycle (NEDC). The engine speed $\omega$ and the drive train torque $\tau_{d}$ for this driving cycle are 
TABLE I

PARAMETERS FOR THE DRIVE TRAIN MODEL

$\begin{array}{llll}\text { Quantity } & \text { Symbol } & \text { Value } & \text { Unit } \\ \text { Mass } & \mathrm{m} & 1400 & \mathrm{~kg} \\ \text { Frontal area } & A_{d} & 2 & \mathrm{~m}^{2} \\ \text { Air drag coefficient } & C_{d} & 0.3 & - \\ \text { Rolling resistance } & C_{r} & 0.015 & - \\ \text { Air density } & \rho & 1.2 & \mathrm{~kg} / \mathrm{m}^{3} \\ \text { Gravity } & g & 9.8 & \mathrm{~m} / \mathrm{s}^{2} \\ \text { Wheel radius } & w_{r} & 0.3 & \mathrm{~m} \\ \text { Final drive ratio } & f_{r} & 4.0 & - \\ \text { Gear ratio } & g_{r} & 3.4-2.1-1.4-1.0-0.77 & -\end{array}$

calculated using the following formulas:

$$
\begin{aligned}
\omega(t) & =\frac{f_{r}}{w_{r}} g_{r}(t) v(t) \\
\tau_{d}(t) & =\frac{w_{r}}{f_{r}} \frac{1}{g_{r}(t)} F_{d}(t) \\
F_{d}(t) & =m \dot{v}(t)+\frac{1}{2} \rho C_{d} A_{d} v(t)^{2}+m g C_{r}
\end{aligned}
$$

The actual parameter values and their physical meaning are given in Table I. At moments that the drive train requests negative torque, this is provided by the engine (which has a negative drag-torque) and the alternator (by recovering free kinetic energy). The mechanical brakes are only used when the engine and the alternator together do not achieve the requested vehicle deceleration. The alternator can provide electric power up to $1.6 \mathrm{~kW}$ and the $12 \mathrm{~V}$ battery has a capacity of 50Ah. The effective capacity is assumed to be $E_{c a p}=10^{6} \mathrm{~J}$ with the efficiency parameters $\eta=0.9$, $b^{-}=b^{+}=\sqrt{\eta}$ and $\beta=5 \times 10^{-5}$. Simulations always start at $70 \%$ SOC.

\section{B. Evaluated strategies}

Simulation results of the following three strategies will be analyzed:

$B L x \quad$ Baseline strategy where the alternator always provides the requested power for the electric loads.

$M P C x$ Optimization strategy in MPC-framework; Exact prediction with horizon length $N_{p}$ is assumed.

$R B x \quad$ Rule-based strategy as defined in (32) and (33).

The index term $x$ behind each strategy name indicates the selected electric load profile, see Table II. The second and third column in Table II represent the constraint in (12). The fourth column defines the function $E_{L \text { min }}(k)$ from (26). To be able to compare different strategies, an average electric load of 250W is selected in BL1, MPC0, MPC1 and RB1. The profiles BL2, MPC2 and RB2 supply 500W to the loads.

\section{Simulation results}

The influence of the prediction horizon length is evaluated for MPC0 and MPC1. Fig. 3a shows the reduction in fuel consumption whereas Fig. $3 \mathrm{~b}$ visualizes the total amount of energy stored in the battery. Both strategies are simulated for a receding horizon with $N_{p}=1, . ., 1181$ and $\Delta T=1 \mathrm{~s}$. When the prediction horizon reaches the end of the driving cycle, all remaining control actions are directly taken from
TABLE II

SPECIFICATION OF Electric LoAd PRofiLes

$\begin{array}{llcc}\text { Profile } & P_{L \min }[\mathrm{W}] & P_{L \max }[\mathrm{W}] & E_{L \min }[\mathrm{J}] \\ \text { BL1 } & 250 & 250 & - \\ \text { BL2 } & 500 & 500 & - \\ \text { MPC0 } & 250 & 250 & 250 \times k \\ \text { MPC1 } & 0 & 500 & 250 \times k \\ \text { MPC2 } & 250 & 750 & 500 \times k \\ \text { RB1 } & 0 \text { if } E_{L}(t) \geq 250 t & 500 & - \\ & 250 \text { if } E_{L}(t)<250 t & & - \\ \text { RB2 } & 250 \text { if } E_{L}(t) \geq 500 t & 750 & - \\ & 500 \text { if } E_{L}(t)<500 t & & \end{array}$
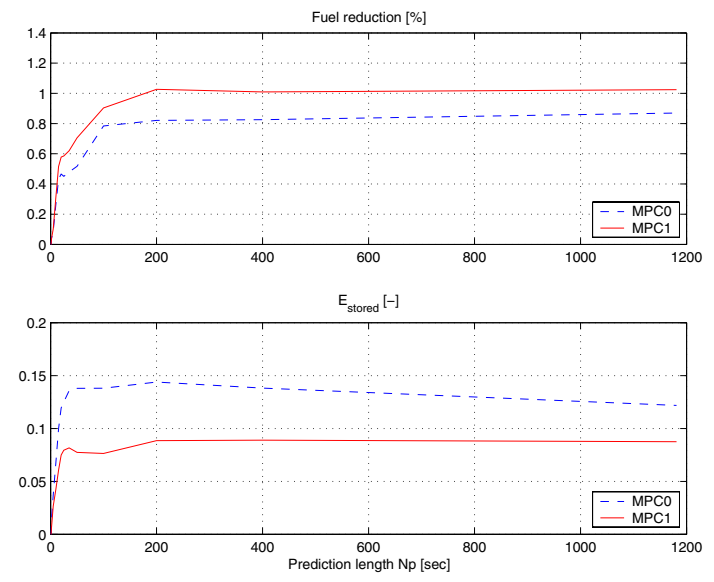

Fig. 3. Influence of prediction horizon on (a) fuel use and (b) battery use

that time instant. The results in Fig. $3 \mathrm{~b}$ are normalized with respect to the capacity of the battery:

$$
\mathcal{E}_{\text {stored }}=\frac{\int_{0}^{t_{e}} P_{b}(t) d t}{E_{\text {cap }}} \quad \text { for } \quad P_{b}(t) \geq 0
$$

Fig. 4 visualizes the control sequence for the MPC1 profile with $N_{p}=1181$ (dashed curve) as well as for the RB1 profile (solid curve) over the last 600s of the NEDC driving cycle. Especially during regenerative braking phases, corresponding control actions for $P_{b}$ and $P_{L}$ can be observed.

Table III summarizes the main characteristics of each strategy. Again, MPCx uses $N_{p}=1181$. The second column considers the relative amount of fuel necessary for only supplying the electric loads. The third column compares the overall fuel consumption of the MPC and RB profile to the corresponding BL profile. Finally, the fourth column indicates the total amount of energy stored in the battery, as calculated with (37).

\section{Evaluation}

Although the profits in fuel reduction are limited, they increase rapidly until $N_{p}=200$ and remain almost constant for a higher prediction length, see Fig. 3a. This behavior can be explained as follows. The end-point constraint in (24) is very dominant for short predictions, resulting in a very small operating window for the battery. Moreover, the desired vehicle speed has a large influence on the operating 

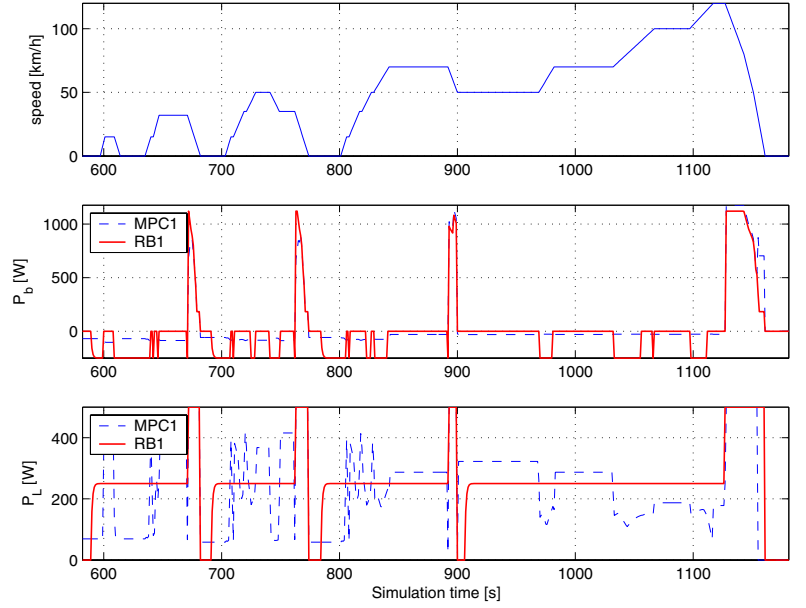

Fig. 4. Control sequence for MPC1 and RB1 profile

TABLE III

STRATEGY RESULTS

$\begin{array}{lccc}\text { Profile } & \begin{array}{c}\text { Relative costs } \\ \text { electr. power }[\%]\end{array} & \begin{array}{c}\text { Overall reduction } \\ \text { in fuel usage [\%] }\end{array} & \begin{array}{c}\text { Normalized } \\ \text { bat. usage }[-]\end{array} \\ \text { BL1 } & 100 & 0.00 & 0.000 \\ \text { MPC0 } & 74.3 & 0.87 & 0.122 \\ \text { MPC1 } & 69.7 & 1.02 & 0.088 \\ \text { RB1 } & 70.3 & 1.00 & 0.091 \\ \text { BL2 } & 100 & 0.00 & 0.000 \\ \text { MPC2 } & 90.1 & 0.65 & 0.086 \\ \text { RB2 } & 89.1 & 0.71 & 0.113\end{array}$

point of the combustion engine and consequently also the incremental cost $\lambda$ as defined in (27). As shown in [4], the frequency spectrum of $\lambda$ gives insight into the minimum length of the prediction horizon, so the speed profile of the NEDC is closely related to the required prediction length.

From the results in Fig. 3a and Fig. 3b, one can deduce that the loads with a flexible power demand in MPC1 (solid curve) contribute to a reduction in fuel use of $18 \%$, whereas the battery activity decreases at least $28 \%$. Therefore, it is recommended to apply loads with a flexible power demand if possible.

Considering Fig. 4b and Fig. 4c, the MPC1 strategy shows smooth switching behavior for the battery power $P_{b}$, whereas the load power $P_{L}$ can switch at high frequencies. Such behavior for $P_{L}$ was expected because this variable has only been included in the constraints and not in the cost criterion. Smooth switching behavior by the RB1 strategy can be seen for $P_{L}$ but not for $P_{b}$. For this strategy, the alternator power $P_{e}$ is calculated by considering the incremental cost at the present moment. This quantity changes rapidly during vehicle acceleration or braking moments, so for a given $P_{L}$ the battery will have to follow the alternator power.

The performance of each strategy is summarized in Table III. The second column shows that the costs for generating electric power can be reduced significantly, although profits are lower for higher load power. There are two reasons for this. First, free energy from regenerative braking is captured by the MPC and RB strategy. Also the BL strategy captures some of this energy and this is more for higher load powers. Second, the power limitations of the alternator become more dominant for higher load powers. This means that the feasible area of the MPC and RB strategy reduces when the load power increases. Note that RB2 performs slightly better than MPC2. This is explained by the fact that the MPC strategy assumes signals to remain constant within each sample interval $\Delta T$, whereas the RB2 strategy continuously updates its control law. Column three in Table III presents the actual benefits in fuel consumption from EM. The profits are limited because the selected vehicle configuration requires relatively less power for the electric loads, compared to the propulsion power. Better performance can be expected from other vehicle configurations (e.g. an HEV), where the electric power demand represents a large part of the total energy consumption. The fourth column again emphasizes that the application of loads with a flexible power demand reduces the battery activity in combination with an EM strategy. Note that RB2 causes relatively much battery activity, compared to MPC2.

\section{CONCLUSIONS AND DISCUSSION}

This paper presents two EM strategies to control the power flow at the electric power net in road vehicles. In the analytical MPC approach, a prediction of the future driving cycle is required, whereas the rule-based strategy only relies on vehicle information up to the present moment. Simulations showed that both concepts can yield almost the same performance. Nevertheless, fuel savings are limited here due to the selected vehicle configuration.

More freedom in control is achieved by introducing electric loads with a flexible power demand. This extra freedom results in a further reduction in fuel consumption. Moreover, a significant decrease in battery activity can be noticed, making these flexible loads perfectly suitable for EM.

\section{REFERENCES}

[1] M. Åsbogård, F. Edström, J. Bringhed, M. Larsson, and J. Hellgren. Evaluating potential of vehicle auxiliary system coordination using optimal control. In Proc. of the $7^{\text {th }}$ Int. Symp. on Advanced Vehicle Control (AVEC), Arnhem, The Netherlands, August 2004.

[2] M. Back, M. Simons, F. Kirschaum, and V. Krebs. Predictive control of drivetrains. In Proc. of the IFAC 15th Triennial World Congress, Barcelona, Spain, 2002.

[3] B. de Jager. Predictive storage control for a class of power conversion systems. In Proc. of the European Control Conf., Cambridge, UK, September 2003.

[4] B. de Jager. Choosing the horizon in predictive storage control. In Proc. of the American Control Conf., Boston, USA, June 2004.

[5] M. Koot, J. Kessels, B. de Jager, M. Heemels, P. van den Bosch, and M. Steinbuch. Energy management strategies for vehicular electric power systems. IEEE Trans. on Vehicular Technology, 54(3), May 2005.

[6] C.-C. Lin, H. Peng, J.W. Grizzle, and J.-M. Kang. Power management strategy for a parallel hybrid electric truck. IEEE Trans. on Control Systems Technology, 11(6):839-849, November 2003.

[7] G. Paganelli, G. Ercole, A. Brahma, Y. Guezennec, and G. Rizzoni. General supervisory control policy for the energy optimization of charge-sustaining hybrid electric vehicles. JSAE Review, 22(4):511518, April 2001.

[8] A. Sciarretta, M. Back, and L. Guzzella. Optimal control of parallel hybrid electric vehicles. IEEE Trans. on Control Systems Technology, 12(3):352-362, May 2004.

[9] E.D. Tate and S.P. Boyd. Finding ultimate limits of performance for hybrid electric vehicles. SAE Paper 2000-01-3099. 